\title{
Melatonin alleviates liver steatosis induced by prenatal dexamethasone exposure and postnatal high-fat diet
}

\author{
CHING-CHOU TSAI ${ }^{1,2}$, YU-JU LIN ${ }^{1}$, HONG-REN YU ${ }^{3}$, JIUNN-MING SHEEN ${ }^{3}$, \\ YOU-LIN TAIN ${ }^{3,4}$, LI-TUNG HUANG ${ }^{3 *}$ and MAO-MENG TIAO ${ }^{3 *}$
}

\author{
${ }^{1}$ Department of Obstetrics and Gynecology, Kaohsiung Chang Gung Memorial Hospital, Chang Gung University, \\ College of Medicine, Kaohsiung 83301; ${ }^{2}$ Department of Obstetrics and Gynecology, Chiayi Chang Gung Memorial Hospital, \\ Chiayi 61363; ${ }^{3}$ Department of Pediatrics, Kaohsiung Chang Gung Memorial Hospital, Chang Gung University, \\ College of Medicine; ${ }^{4}$ Institute for Translational Research in Biomedicine, Kaohsiung Chang Gung Memorial Hospital, \\ Chang Gung University, College of Medicine, Kaohsiung 83301, Taiwan, R.O.C.
}

Received January 23, 2017; Accepted September 13, 2017

DOI: $10.3892 /$ etm.2018.6256

\begin{abstract}
Prenatal exposure to glucocorticoids is associated with negative health consequences for the offspring that persist into adulthood, including liver steatosis. Melatonin has previously been demonstrated to suppress liver steatosis and oxidative stress in humans with non-alcoholic fatty liver disease and in animal models of obesity. The present study aimed to determine whether melatonin protects against liver steatosis induced by prenatal dexamethasone exposure followed by postnatal high-fat diet. Pregnant Sprague-Dawley rats at gestational days 14-21 were administered dexamethasone $(0.1 \mathrm{mg} / \mathrm{kg} / \mathrm{day})$ or saline via intraperitoneal injection. The offspring were then divided into five groups, as follows: Vehicle, postnatal high-fat diet (VHF), prenatal dexamethasone exposure (DEX), prenatal dexamethasone exposure + postnatal high-fat diet (DHF), and prenatal dexamethasone exposure + postnatal high-fat diet + melatonin (DHFM) group. Following vehicle or dexamethasone exposure of the maternal rats, the offspring rats in the VHF, DHF and DHFM groups received a high-fat diet (58\% fat) between weaning and 6 months of age. In the DHFM group,
\end{abstract}

Correspondence to: Professor Mao-Meng Tiao or Professor Li-Tung Huang, Department of Pediatrics, Kaohsiung Chang Gung Memorial Hospital, Chang Gung University, College of Medicine, 123 Ta-Pei Road, Niao Sung, Kaohsiung 83301, Taiwan, R.O.C.

E-mail: tmm@cgmh.org.tw

E-mail: litung.huang@gmail.com

${ }^{*}$ Contributed equally

Abbreviations: NAFLD, non-alcoholic fatty liver disease; TUNEL, TdT-mediated dUTP-biotin nick end-labeling; SOCS3, suppressor of cytokine signaling 3; MDA, malondialdehyde; MnSOD, manganese superoxide dismutase; PI3K, phosphoinositide 3-kinase

Key words: high-fat diet, liver steatosis, dexamethasone, melatonin, obesity melatonin was administered to the mothers from gestational days 14-21 until weaning. The offspring continued to receive melatonin until they were sacrificed at 6 months old. Oil Red $\mathrm{O}$ staining demonstrated stronger intensity in the DHF group compared with that in the other four groups. Western blot analysis also revealed higher levels of cleaved caspase-3, tumor necrosis factor- $\alpha$ (TNF- $\alpha)$, suppressor of cytokine signaling 3 (SOCS3) and malondialdehyde (MDA), as well as reduced expression of manganese superoxide dismutase (MnSOD) and phosphoinositide 3-kinase (PI3K) in the DHF group compared with the vehicle and DHFM groups. In addition, melatonin reduced the Oil Red $\mathrm{O}$ staining intensity and the levels of cleaved caspase-3, TNF- $\alpha$, SOCS3 and MDA, while it increased the MnSOD and PI3K levels, in the DHFM group compared with the DHF group. In conclusion, postnatal high-fat diet aggravated the prenatal dexamethasone-induced liver steatosis in adult rat offspring via inflammation, oxidative stress and cellular apoptosis, which may be ameliorated by prenatal melatonin therapy.

\section{Introduction}

Obesity is a worldwide epidemic and the fifth leading cause of mortality (1). Obesity is also a well-documented risk factor for non-alcoholic fatty liver disease (NAFLD), which occurs in approximately one third of the population in the developed countries (2). Liver steatosis, which is common in NAFLD patients, is a chronic liver disease characterized by a spectrum of hepatic pathologies that can lead to cirrhosis (3).

Pregnant women may experience preterm delivery, and $\sim 7 \%$ of them are treated with synthetic glucocorticoids to improve neonatal outcomes. However, prenatal exposure to glucocorticoids has been reported to be associated with negative health consequences for the offspring that persist into adulthood (4,5). It has become increasingly clear that early environmental influences also have a long-lasting impact on the development and physiology of the fetus. For instance, prenatal glucocorticoid overexposure may result in liver steatosis (6). Previous studies have observed that prenatal exposure to dexamethasone increased lipid accumulation in 
the liver in rats on a high-fat diet, while the deleterious effects of the high-fat diet in the perinatal and post-weaning periods persisted into adulthood $(7,8)$. The present study aimed to expand upon the results of a previous study by our group, which also investigated the effect of melatonin (9). In addition, our previous study identified that postnatal high-fat diet increased liver steatosis and apoptosis exacerbated by prenatal dexamethasone exposure via an oxidative effect (6). High-fat diet is an environmental risk factor for disease progression in patients with NAFLD subsequent to prenatal stress. Thus, it is important to identify a strategy to prevent liver steatosis in the perinatal period.

Melatonin, an indoleamine secreted from the pineal gland, has a wide range of physiological and pharmacological functions, as well as beneficial effects in metabolic diseases (10-12). The administration of melatonin reduced the metabolic pathologies associated with the intake of high-fat diet, suggesting its protective role (13). A further animal study demonstrated the therapeutic amelioration caused by melatonin, which improved the metabolic syndrome induced by high fructose intake in rats (14). In addition, melatonin maintains the biological membrane fluidity, and functions as an antioxidant and reactive oxygen species scavenger at the mitochondrial level (15). Melatonin also counteracts adipogenesis by stimulating thermogenesis, insulin sensitivity and glucose uptake, as well as by improving the liver function in different metabolic and physiological conditions (16). Furthermore, the hepatoprotective effect of melatonin in suppressing steatosis and oxidative stress has been observed in human patients with NAFLD (17) and in animal models of obesity (18). Certain investigators observed that the use of pentoxifylline and melatonin in combination with pioglitazone ameliorated experimental NAFLD, thus indicating that pentoxifylline and melatonin can be used as adjuvant therapies in the clinical management of NAFLD (19). Finally, it was previously observed that melatonin alleviated liver steatosis and oxidative injury (8). However, its roles and mechanisms of action are not fully understood in the context of environmental exposure in the perinatal period.

The aim of the present study was to determine whether melatonin protects the liver from NAFDL induced by prenatal dexamethasone exposure and postnatal high-fat diet. The study focused on the investigation of the liver morphology, redox state, apoptosis and metabolic markers.

\section{Materials and methods}

Animals. The present study followed the Guide for the Care and Use of Laboratory Animals of the National Institutes of Health (version 8) (20). The protocol was approved by the Institutional Animal Care and Use Committee of the Kaohsiung Chang Gung Memorial Hospital (Kaohsiung, Taiwan). A total of 15 Sprague-Dawley rats (12-16 weeks old; 200-250 mg; male:female, 1:2; BioLasco Taiwan Co., Ltd., Taipei, Taiwan) were housed in the animal care facility of the Kaohsiung Chang Gung Memorial Hospital at $22^{\circ} \mathrm{C}$ and a $12 \mathrm{~h}$ light/dark cycle (light from 7 a.m.). Pregnant rats were checked for litters daily at 10 a.m. Sprague-Dawley female rats were allowed to mate with male rats for $24 \mathrm{~h}$. After 1 day, female rats were separated from the male rats and housed individually in a standard plastic home cage. Following confirmation of pregnancy on day 14 after mating, pregnant females were randomly subjected to prenatal steroid exposure or left undisturbed until delivery. The day of birth was designated as postnatal day 0 (PND 0). Rat pups were weaned at PND 21 and had free access to standard chow and water.

Grouping. The offspring rats were divided into five groups ( $n=6 /$ group), as follows: Vehicle group, vehicle + postnatal high-fat diet group (VHF), prenatal dexamethasone exposure group (DEX), prenatal dexamethasone exposure + postnatal high-fat diet group (DHF), and prenatal dexamethasone exposure + postnatal high-fat diet + melatonin group (DHFM).

Postnatal high fat diet. Offspring rats in the VHF, DHF and DHFM groups received a 58\% high-fat diet (D12331; Research Diet, Inc., New Brunswick, NJ, USA) from weaning to 6 months of age. The vehicle and DEX groups received control diet, consisting of $23.5 \%$ protein, $4.5 \%$ fat, $5.0 \%$ crude fiber, $7.0 \%$ crude ash and $13 \%$ water (Taiwan Fwusow Industry Co., Ltd., Taichung, Taiwan).

Prenatal dexamethasone or vehicle exposure. Pregnant Sprague-Dawley rats at gestational days 14-21 were administered dexamethasone $(0.1 \mathrm{mg} / \mathrm{kg} /$ day; D4902; Sigma-Aldrich; Merck KGaA, Darmstadt, Germany) or normal saline via intraperitoneal injection in the DEX and vehicle groups, respectively, as reported in a previous study (21).

Melatonin administration. Melatonin was prepared three times a week by dissolving $16 \mathrm{mg}$ dry melatonin in $100 \%$ ethanol (1 ml; M5250; Sigma-Aldrich; Merck KGaA), followed by dilution with distilled water to a final concentration of $40 \mathrm{mg} / \mathrm{l}(0.01 \%)$. The bottles were covered with aluminum foil to protect them from light. Rats were estimated to consume $\sim 25 \mathrm{ml}$ of water per day; thus the mean daily intake of melatonin to the rat mothers in the DHFM group was estimated to be $1 \mathrm{mg} / \mathrm{kg}$ /day from gestational days 14-21 until weaning ended and to the offspring rats in the DHFM group was estimated to be $1 \mathrm{mg} / \mathrm{kg}$ /day until they were sacrificed, as previously reported (6). This melatonin regimen has previously been used in our laboratory, and rats have demonstrated good compliance with beneficial results $(6,22,23)$.

Sacrifice and sample collection. Animals in all five groups were sacrificed at PND 180 by intramuscular injection of ketamine (10 mg/kg; Pfizer, Hsinchu, Taiwan), with efforts made to minimize suffering. Immediately after sacrificing, the liver was resected, weighed, embedded in paraffin and stored at $-80^{\circ} \mathrm{C}$. A blood sample was also collected by cardiocentesis and placed into an EDTA-containing vial. The levels of cholesterol, aspartate aminotransferase (AST) and alanine aminotransferase (ALT) levels in the blood were determined using a standard autoanalyzer (Hitachi 7450; Hitachi, Ltd., Tokyo, Japan).

Immunohistochemical localization of Oil Red O. Frozen liver samples obtained from 6 rats in each group were cut into 2-3- $\mu \mathrm{m}$-thick sections, which were mounted on coating slides. Tissue sections were then incubated with $3 \%$ hydrogen peroxide for $10 \mathrm{~min}$ to block any endogenous peroxidase activity. Next, the sections were boiled in citrate buffer in 
Table I. Weight and biochemical parameters of animals in the experimental groups.

\begin{tabular}{|c|c|c|c|c|c|}
\hline Parameter & Vehicle & VHF & DEX & DHF & DHFM \\
\hline Weight (mg) & $623.0 \pm 17.1^{\mathrm{a}}$ & $759.0 \pm 17.9^{\mathrm{b}}$ & $658.2 \pm 20.0^{\mathrm{a}}$ & $804.4 \pm 35.1^{b, c}$ & $699.2 \pm 13.9^{\mathrm{a}}$ \\
\hline Liver weight (mg) & $17.5 \pm 0.5$ & $18.9 \pm 0.4$ & $20.7 \pm 0.8^{\mathrm{b}}$ & $20.3 \pm 0.9$ & $18.7 \pm 0.2$ \\
\hline Liver/body (\%) & $2.63 \pm 0.1^{\mathrm{c}}$ & $2.48 \pm 0.1^{\mathrm{c}}$ & $3.09 \pm 0.1^{\mathrm{a}, \mathrm{b}}$ & $2.52 \pm 0.1^{\mathrm{c}}$ & $2.68 \pm 0.1^{\mathrm{c}}$ \\
\hline $\operatorname{AST}(\mathrm{U} / \mathrm{l})$ & $95.0 \pm 9.0^{\mathrm{a}}$ & $148.3 \pm 7.1^{\mathrm{a}}$ & $128.9 \pm 19.5^{\mathrm{a}}$ & $413.1 \pm 64.2^{\mathrm{b}, \mathrm{c}}$ & $163.8 \pm 17.7^{\mathrm{a}}$ \\
\hline $\operatorname{ALT}(\mathrm{U} / \mathrm{l})$ & $35.8 \pm 1.8^{\mathrm{a}}$ & $76.0 \pm 8.3^{\mathrm{a}}$ & $57.2 \pm 10.5^{\mathrm{a}}$ & $336.1 \pm 67.2^{\mathrm{b}, \mathrm{c}}$ & $90.2 \pm 10.2^{\mathrm{a}}$ \\
\hline Cholesterol (mg/dl) & $74.3 \pm 2.6^{\mathrm{c}}$ & $79.3 \pm 4.0$ & $102.9 \pm 8.6^{\mathrm{b}}$ & $88.2 \pm 7.1$ & $59.8 \pm 6.8^{a, c}$ \\
\hline
\end{tabular}

${ }^{\mathrm{a}} \mathrm{P}<0.05$ vs. DHF-treated group; ${ }^{\mathrm{b}} \mathrm{P}<0.05$ vs. vehicle-treated group; ${ }^{\mathrm{c}} \mathrm{P}<0.05$ vs. DEX-treated group. Data are expressed as the mean \pm standard error. AST, aspartate transaminase; ALT, alanine aminotransferase; VHF, postnatal high-fat diet group; DEX, prenatal dexamethasone exposure group; DHF, prenatal dexamethasone exposure + postnatal high-fat diet group; DHFM, prenatal dexamethasone exposure + postnatal high-fat diet + melatonin group .

the microwave for 12 min for antigen retrieval, stained with Oil Red O (cat. no. 1.02419; EMD Millipore, Billerica, MA, USA), dissolved in $60 \%$ isopropanol for $15 \mathrm{~min}$ at room temperature, and rinsed in $60 \%$ isopropanol and washing in $\mathrm{dH}_{2} \mathrm{O}$. The tissue was then counterstained by hematoxylin, washed thoroughly in $\mathrm{dH}_{2} \mathrm{O}$ and transferred to Aqua-Mount mounting medium (Thermo Fisher Scientific, Inc., Waltham, MA, USA). The labeling index of hepatocytes was defined as the mean \pm standard error of number of positively stained nuclei among 500 hepatocytes in the liver section. Sections containing at least three portal tracts were used for the labeling index in at least three non-overlapping fields under a light microscope (magnification, x100).

Western blot analysis. Liver tissues from the five rat groups were dissected and frozen immediately in liquid $\mathrm{N}_{2}$. The tissue was homogenized in lysis buffer (cat. no. 17081; iNtRON Biotechnology, Seongnam, Korea) and then centrifuged at $14,000 \mathrm{x} \mathrm{g}$ at $4^{\circ} \mathrm{C}$ for $5 \mathrm{~min}$. Total protein concentration was detected by Bio-Rad Protein Assay Dye Reagent Concentrate (Bio-Rad Laboratories, Inc., Hercules, CA, USA). Protein $(40 \mu \mathrm{g})$ from the supernatant of each sample was separated by $10-15 \%$ SDS-PAGE and transferred to polyvinylidene difluoride membranes by electrophoresis. Next, the membranes were blocked in Tris-buffered saline/Tween-20 buffer containing 5\% low-fat milk powder for $1 \mathrm{~h}$ at room temperature. Immunoblotting was then performed by incubation at $4^{\circ} \mathrm{C}$ for overnight with specific primary antibodies. The monoclonal primary antibodies included cleaved caspase-3 (cat. no. 9661; Cell Signaling Technology, Inc., Danvers, MA, USA), tumor necrosis factor- $\alpha$ (TNF- $\alpha$; cat. no. 3707; Cell Signaling Technology, Inc.), suppressor of cytokine signaling 3 (SOCS3; cat. no. ab16030; Abcam, Cambridge, MA, USA), phosphoinositide 3-kinase (PI3K; cat. no. ab40755; Abcam), antioxidant manganese superoxide dismutase (MnSOD; cat. no. sc133134; Santa Cruz Biotechnology, Inc., Dallas, TX, USA), malondialdehyde (MDA; ab27642; Abcam), and the internal control, GAPDH (cat. no. ab9485; Abcam). The membranes were then incubated for $1 \mathrm{~h}$ at room temperature with an alkaline phosphatase (AP) conjugated anti-rabbit (1:5,000; cat. no. S3731; Promega Corp., Madison, WI, USA) or mouse (1:10,000; cat. no. 715-055-150; Jackson
ImmunoResearch Laboratories, Inc., West Grove, PA, USA) secondary antibody $(6,24,25)$. Subsequently, the western blots were visualized using the ProtoBlot II AP System (Promega Corp.), The western blotting results were quantified by the colorimetric method using Quantity One software (version 4.5.2; Bio-Rad Laboratories, Inc.).

Terminal deoxynucleotidyl transferase dUTP nick end labeling (TUNEL) assay. In order to investigate cellular apoptosis in the liver tissue, TUNEL staining was performed as previously reported (26). ApopTag Plus Peroxidase in situ Apoptosis Detection kit (Chemicon; EMD Millipore) was used for TUNEL assay. Briefly, deparaffinized sections obtained from 6 rats in each group were washed with distilled water and treated with a protease for $15 \mathrm{~min}$ at $37^{\circ} \mathrm{C}$. Sections containing at least three portal tracts were used for counting the labeling index in at least three non-overlapping fields under a microscope, and the mean \pm standard error of these counts was considered as a labeling index. A total of 500 hepatocytes in each rat were used to count positively stained cells.

Statistical analysis. SPSS software (version 15.0; SPSS, Inc., Chicago, IL, USA) was used for statistical analysis. Statistical analysis was conducted using analysis of variance with a Bonferroni post hoc test. The data are presented as the mean \pm standard error of the mean. Statistically significant differences were indicated by values of $\mathrm{P}<0.05$.

\section{Results}

AST and ALT levels, and the body weight of the rats are increased in the DHF group and decreased in the DHFM group. As demonstrated in Table I, the body weight was significantly higher in the DHF group as compared with that in the DHFM and DEX groups. There was no significant difference in the liver weight among the DHF, vehicle and DHFM groups. By contrast, rats in the DHF group exhibited a higher body weight and a lower liver/body ratio compared with the DEX group. In addition, the DHF group had a significantly lower liver/body ratio comparing with the DEX group, but no significant difference when compared with the Vehicle, VHF and DHFM groups. 


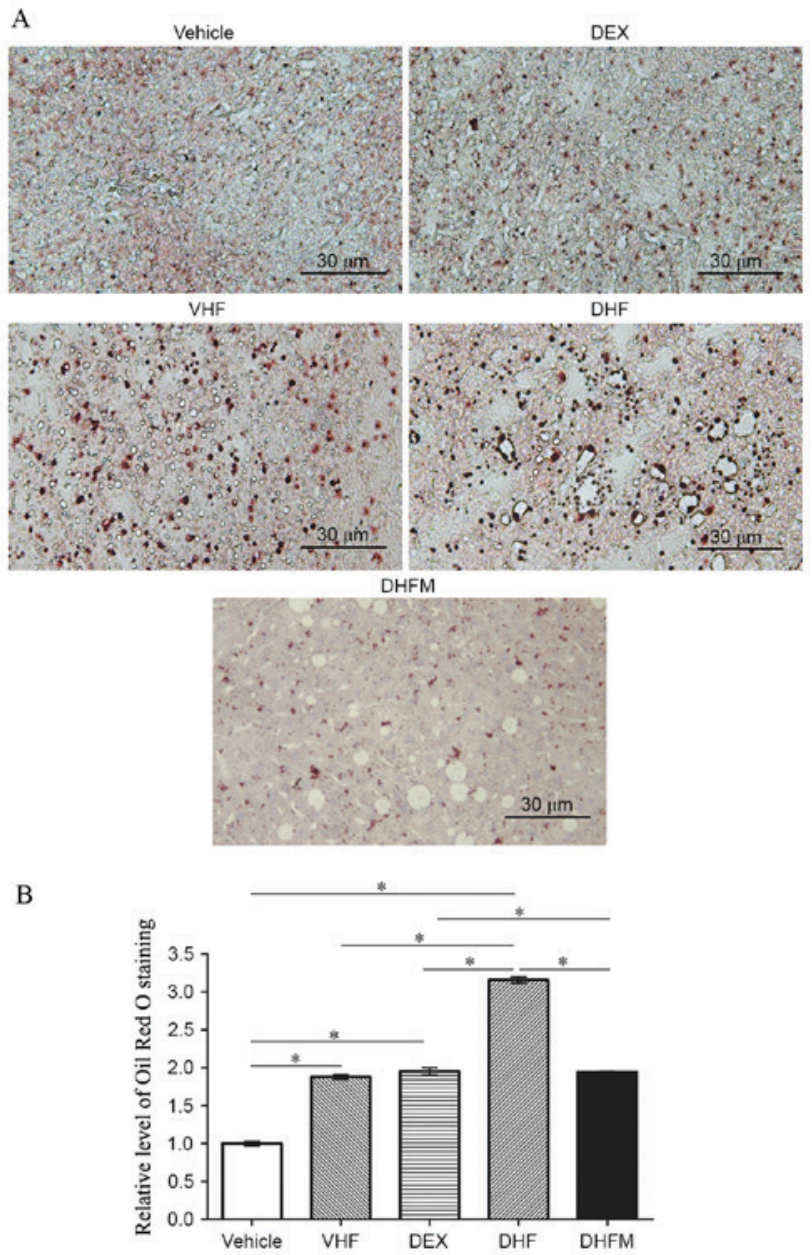

Figure 1. Liver steatosis assessed by Oil Red O staining. Sections were obtained from 6 rats in each group. Following formalin fixation, these paraffin-embedded liver sections were stained and evaluated. (A) Stronger Oil Red O staining intensity was observed in the DHF group, which was then reduced subsequent to melatonin administration (magnification, x400; scale bar, $30 \mu \mathrm{m}$ ). (B) Semi-quantitative analysis of Oil Red O stained cells. All values are presented as the mean \pm standard error $(n=6) .{ }^{*} \mathrm{P}<0.05$. VHF, postnatal high-fat diet; DEX, prenatal dexamethasone; DHF, prenatal dexamethasone + postnatal high-fat diet; DHFM, prenatal dexamethasone + postnatal high-fat diet + melatonin

Animals in the DHF group presented the highest AST and ALT levels (Table I). Furthermore, the levels of AST, ALT and cholesterol were significantly reduced in the DHFM group compared with the DHF group. The cholesterol level in the DHF group was not statistically significant compared with the Vehicle, VHF, DEX and DHFM groups (Table I).

Liver steatosis in the DHFM group. Oil Red O staining of the liver tissues (Fig. 1A) exhibited a stronger intensity in the DHF group compared with that in the other four groups, indicating a synergistic effect between prenatal dexamethasone exposure and postnatal high-fat diet. In addition, the results demonstrated that melatonin administration reduced the Oil Red $\mathrm{O}$ staining level in the DHFM group as compared with the DHF group $(\mathrm{P}<0.05$; Fig. 1B). This suggested that melatonin was efficient in reducing the liver lipid storage by attenuating liver steatosis in rats with postnatal high-fat diets and exposed to prenatal dexamethasone.

Apoptosis in the DHFM group. Activation of apoptotic pathways was detected based on the extent of TUNEL
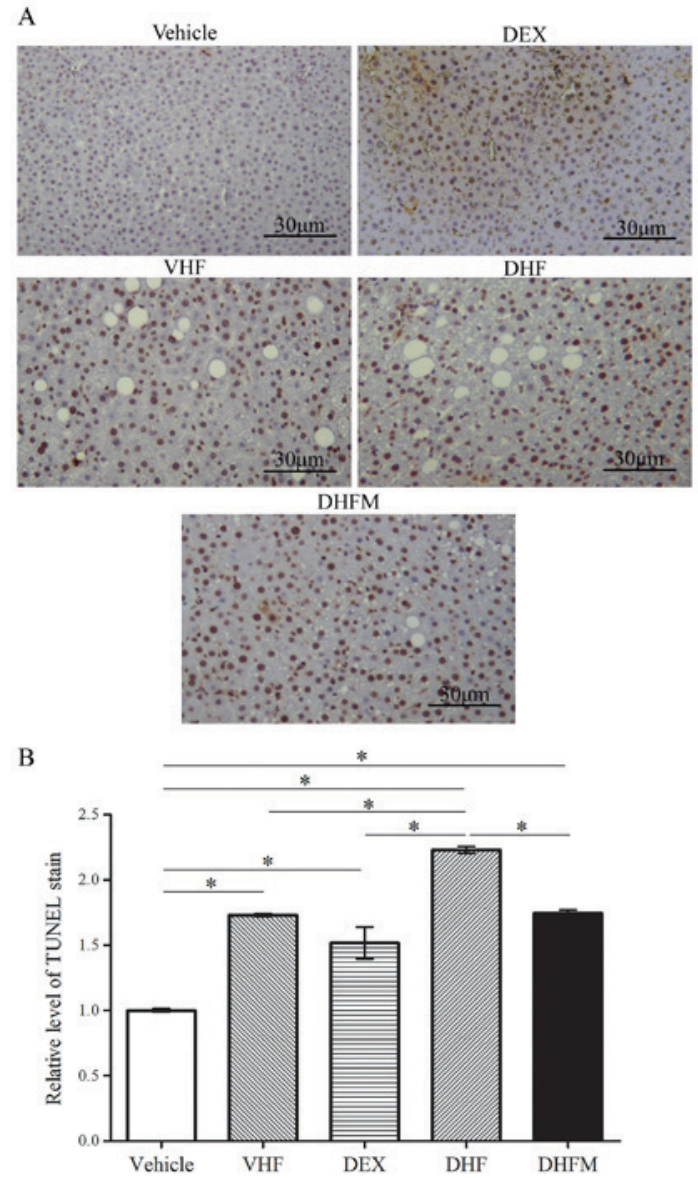

Figure 2. TUNEL assay was conducted to analyze the role of apoptosis in liver damage. (A) TUNEL staining was stronger in the DHF, DEX and VHF groups as compared with the vehicle-treated group, while the DHFM group presented reduced TUNEL staining in comparison with the DHF group (magnification, $\mathrm{x} 400$; bar, $30 \mu \mathrm{m}$ ). (B) Semi-quantitative analysis of TUNEL stained cells. All values are expressed as the mean \pm standard error $(n=6)$. ${ }^{*} \mathrm{P}<0.05$. VHF, postnatal high-fat diet; DEX, prenatal dexamethasone; DHF, prenatal dexamethasone + postnatal high-fat diet; DHFM, prenatal dexamethasone + postnatal high-fat diet + melatonin; TUNEL, TdT-mediated dUTP-biotin nick end-labeling.

staining (Fig. 2), as well as the level of cleaved caspase-3 (Fig. 3A and B). TUNEL staining revealed a significantly greater proportion of apoptotic cells in the DHF group compared with that in the other four groups, indicating a synergistic effect between prenatal dexamethasone exposure and postnatal high-fat diet (Fig. 2). Following melatonin administration to the offspring rats in the DHFM group, the degree of TUNEL staining was decreased in comparison with that in the DHF group $(\mathrm{P}<0.05$; Fig. $2 \mathrm{~B})$. These findings suggested that melatonin was efficient in reducing liver cell apoptosis in rats with prenatal dexamethasone exposure and postnatal high-fat diet.

The level of cleaved caspase-3 was significantly higher in the DHF group as compared with Vehicle and DHFM groups (Fig. 3B). Following melatonin administration, the level in the DHFM group was significantly decreased compared with the DHF group $(\mathrm{P}<0.05)$, suggesting that melatonin was efficient in reducing the level of cleaved caspase- 3 and thus decreasing apoptosis via insulin resistance and oxidative stress in rats subjected to prenatal dexamethasone exposure and postnatal high-fat diet. 


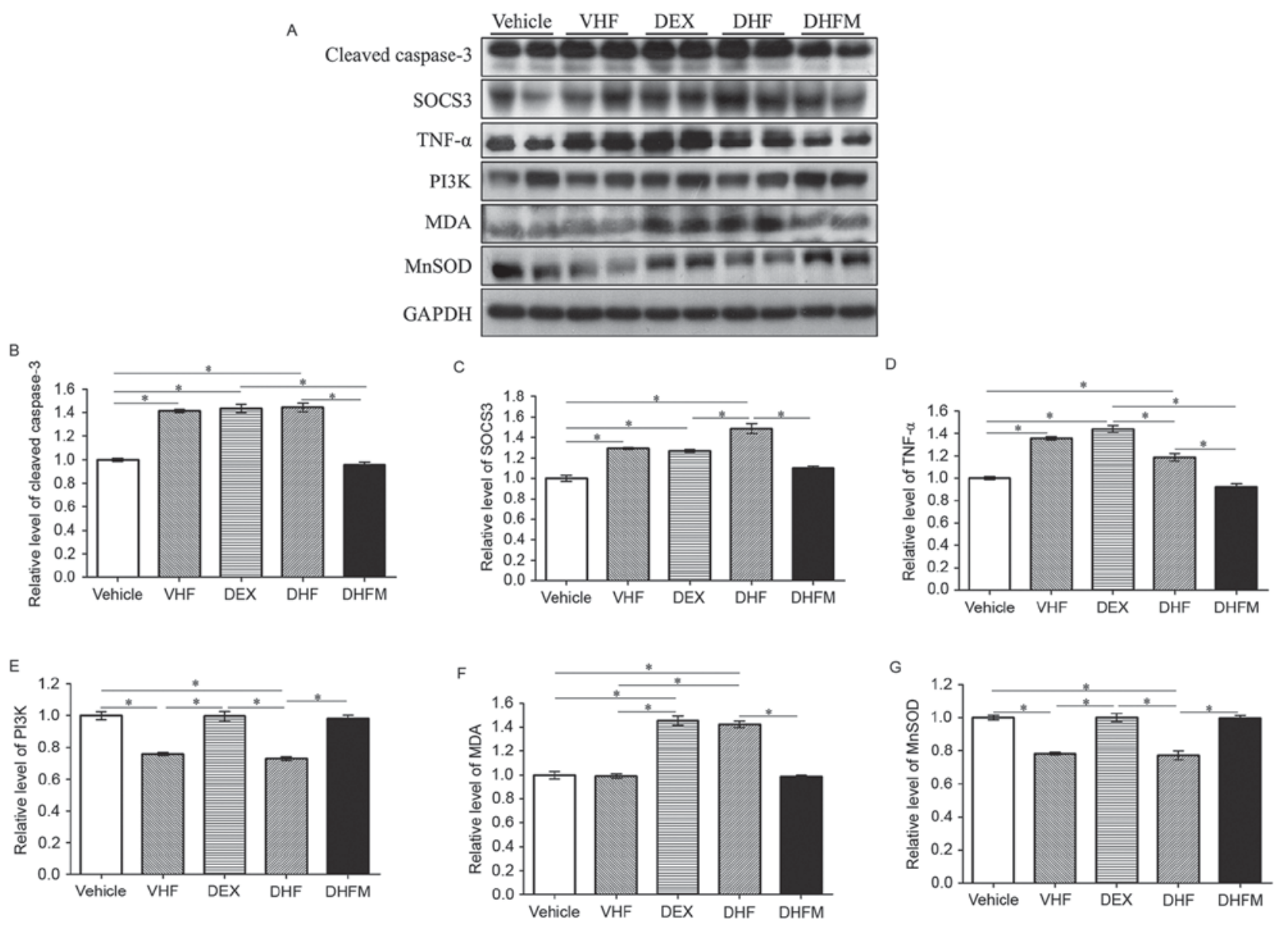

Figure 3. Western blotting and analyses of (A) western blotting results revealed the changes in protein expression in the five groups. Protein expression levels of (B) cleaved caspase-3, (C) SOCS3, (D) TNF- $\alpha$, (E) PI3K, (F) MDA and (G) MnSOD, examined by western blot analysis. Higher expression levels of cleaved caspase-3, TNF- $\alpha$, SOCS3 and MDA were observed in the DHF group and lower levels in the DHFM group. By contrast, the expression of PI3K and MnSOD was decreased in the DHF group compared with that of the DHFM group. All values are expressed as the mean \pm standard error $(\mathrm{n}=6)$. ${ }^{*} \mathrm{P}<0.05$. VHF, postnatal high-fat diet; DEX, prenatal dexamethasone; DHF, prenatal dexamethasone + postnatal high-fat diet; DHFM, prenatal dexamethasone + postnatal high-fat diet + melatonin; SOCS3, suppressor of cytokine signaling 3; MDA, malondialdehyde; TNF- $\alpha$, tumor necrosis factor $\alpha$; MnSOD, manganese superoxide dismutase; PI3K, phosphoinositide-3-kinase.

Insulin resistance and inflammation in the DHFM group. Western blot analysis (Fig. 3A) also revealed significantly higher SOCS3 levels in the DHF group in comparison with that in the DHFM, DEX and Vehicle groups (Fig. 3C). Following melatonin administration, SOCS3 expression was decreased (DHF vs. DHFM groups; $\mathrm{P}<0.05$ ), suggesting that melatonin effectively reduced the SOCS3 protein expression in rats with prenatal dexamethasone exposure and postnatal high-fat diet.

TNF- $\alpha$ is a cell signaling protein involved in systemic inflammation that has been proposed to be associated with insulin resistance (27) and to cause insulin resistance (28). Rats in the DHF group exhibited significantly higher levels of TNF- $\alpha$ compared with animals in the DHFM and Vehicle groups (Fig. 3D). These results suggest that melatonin was efficient in reducing the TNF- $\alpha$ level and this insulin resistance in rats with prenatal dexamethasone exposure and postnatal high-fat diet.

PI3 kinases are key components of the insulin signaling pathway $(29,30)$. PI3 kinases are also associated with oxidative stress (31). Rats in the DHF group demonstrated reduced PI3K protein expression compared with animals in the Vehicle, DEX and DHFM groups (Fig. 3E), indicating a synergistic effect between prenatal dexamethasone exposure and postnatal high-fat diet. Furthermore, melatonin administration increased the PI3K expression (DHF vs. DHFM groups; $\mathrm{P}<0.05$ ), suggesting that melatonin was efficient in restoring the PI3K protein expression in rats with prenatal dexamethasone exposure and postnatal high-fat diet.

Anti-oxidative stress in the DHFM group. MDA results from lipid peroxidation of polyunsaturated fatty acids and is used as a biomarker to measure the level of oxidative stress $(32,33)$. Rats in the DHF group presented higher MDA levels in comparison with animals in the Vehicle, VHF and DHFM groups (Fig. 3F). Subsequent to melatonin administration in DHFM rats, the MDA level was decreased compared with the DHF group $(\mathrm{P}<0.05)$, suggesting that melatonin was efficient in reducing oxidative stress in rats with prenatal dexamethasone exposure and postnatal high-fat diet.

The crucial role of MnSOD in protecting cells against oxidative stress is well known (34). In the present study, rats in the DHF group exhibited a decreased MnSOD level in comparison with that in animals of the other four groups, indicating a synergistic effect between prenatal dexamethasone exposure and postnatal high-fat diet (Fig. 3G). However, melatonin administration increased the MnSOD level 
(DHF vs. DHFM groups; $\mathrm{P}<0.05$ ), suggesting that melatonin was efficient in restoring the MnSOD protein expression, which exhibits the anti-oxidative and protective effects, in rats with prenatal dexamethasone exposure and postnatal high-fat diet. Therefore inflammation was reduced.

Oil Red O staining and western blot analyses of cleaved caspase-3, TNF- $\alpha$, SOCS3, MDA, MnSOD and PI3K demonstrated that their expression levels were similar in the Vehicle and DHFM groups following melatonin administration. In the VHF group, cleaved caspase-3, TNF- $\alpha$, SOCS3 expression levels and TUNEL staining were increased, and PI3K and MnSOD expression levels were decreased compared with the Vehicle group as inflammation and the level of apoptosis were found to be higher in the postnatal high fat diet.

\section{Discussion}

The present study demonstrated that prenatal melatonin administration in rats exposed to dexamethasone prenatally and receiving a high-fat diet postnatally was efficient in: i) Reducing the liver lipid storage; ii) decreasing the expression levels of cleaved caspase- 3 , TNF- $\alpha$, SOCS3 and MDA in the liver; and iii) restoring the liver PI3K and MnSOD protein expression levels in the liver.

Animals in all five groups were sacrificed at PND 180, and body weight measurement indicated a higher weight in the DHF group as compared with that in the DHFM and DEX groups (Table I). However, there was no significant difference in the liver weight among the DHF, vehicle and DHFM groups. In addition, higher body weight and lower liver/body ratio were detected in the DHF group as compared with the DEX group. In the present study, it was also observed that the cholesterol in the DEX group was higher than that in the DHF group, although these groups presented a higher cholesterol level when compared with the vehicle group. These results are similar to the observations of our previous study (9). Additionally, the DHFM group presented the lowest cholesterol level, it was significantly less compared with the DEX and DHF group. Furthermore, animals in the DHF group exhibited the highest AST and ALT levels. The results demonstrate that melatonin treatment led to reduced the levels of AST, ALT and cholesterol.

In our previous study (9), it was observed that rats in the DHF group had stronger liver lipid accumulation as compared with rats in the VHF and DEX groups. Therefore, a high-fat diet in combination with prenatal dexamethasone exposure may lead to more severe lipid accumulation and liver injury. In the present study, melatonin administration reduced the lipid storage in the DHF group. To the best of our knowledge, this is the first study to demonstrate that melatonin administration is able to prevent liver steatosis in adult rats induced by a combination of prenatal dexamethasone exposure and postnatal high-fat diet.

Kupffer cells are major producers of cytokines, modulating the levels of TNF- $\alpha$, and a higher TNF- $\alpha$ expression is correlated with Kupffer cell dysfunction or activation $(35,36)$. Thus, higher TNF- $\alpha$ expression during prenatal dexamethasone administration may indicate inflammation. Decreased TNF- $\alpha$ expression in the DHFM group compared with the DHF group indicates that prenatal melatonin treatment reduced inflammation.
Apoptosis is the main process contributing to disease progression in NAFLD (37). Our previous study revealed increased liver apoptosis in rats with prenatal dexamethasone exposure that were receiving a high-fat diet postnatally (8). In addition, there is growing evidence that melatonin may directly affect the pathways associated with apoptosis (38-40). In the present study, it was demonstrated that melatonin reduced the apoptosis by decreasing the level of cleaved caspase-3 in rats with prenatal dexamethasone exposure and postnatal high-fat diet.

Oxidative stress is another major contributor to disease progression in NAFLD (27), and previous studies indicated that the hepatic MDA level was increased in high-fat diet-induced NAFLD $(41,42)$. Furthermore, MDA levels were increased in rats with NAFLD, and the PI3K level was decreased during oxidative stress in rats on a high-fat diet $(31,43)$. The results of a previous study by our group are consistent with the observations of the present study (9), it revealed that a higher level of oxidative stress in the DHF group was accompanied by increased MDA and decreased MnSOD levels, which was not observed in the DEX group. In addition, the level of PI3K in the DHF group was decreased, suggesting that it participates in the pathogenesis of oxidative stress. In the present study, it was demonstrated that melatonin administration reduced the oxidative stress by lowering the MDA level, as well as increasing the MnSOD and PI3K levels, in rats with prenatal dexamethasone exposure receiving a high-fat diet postnatally.

SOCS3 contributes to leptin and insulin resistance, and certain studies have demonstrated that removal of the SOCS gene prevents insulin resistance in obesity $(44,45)$. A previous study also observed that overexpression of SOCS3 in adipocytes led to a reduction in insulin signaling activation, and diminished the glucose uptake and lipogenesis in mice that were resistant to the development of diet-induced obesity and associated insulin resistance (46). SOCS3 is known to serve two functions in insulin resistance in the liver. Hepatic SOCS3 expression is able to mediate insulin resistance in the liver, whereas the lack of SOCS3 in the liver may stimulate nuclear factor- $\mathrm{kB}$-dependent chronic inflammation, which may also result in systemic insulin resistance $(47,48)$. In the present study, SOCS3 was overexpressed in the liver of animals in the DHF group, therefore insulin resistance was increased, and its level was significantly decreased following melatonin administration. Melatonin may decrease insulin resistance by decreasing SOCS3.

In conclusion, the present study demonstrated that a high-fat postnatal diet exacerbated the effect of prenatal dexamethasone exposure and led to enhanced liver steatosis in adult offspring rats, which was reversed by prenatal melatonin administration.

\section{Acknowledgements}

The present study was supported by grants from the Kaohsiung Chang Gung Memorial Hospital, Kaohsiung, Taiwan (nos. CMRPG8C0841, CMRPG8F0131 and CMRPG8E0641).

\section{References}

1. Malik VS, Willett WC and Hu FB: Global obesity: Trends, risk factors and policy implications. Nat Rev Endocrinol 9: 13-27, 2013. 
2. Cohen JC, Horton JD and Hobbs HH: Human fatty liver disease: Old questions and new insights. Science 332: 1519-1523, 2011.

3. Stacchiotti A, Favero G, Lavazza A, Golic I, Aleksic M, Korac A, Rodella LF and Rezzani R: Hepatic macrosteatosis is partially converted to microsteatosis by melatonin supplementation in $\mathrm{ob} / \mathrm{ob}$ mice non-alcoholic fatty liver disease. PLoS One 11: e0148115, 2016.

4. Kapoor A, Petropoulos S and Matthews SG: Fetal programming of hypothalamic-pituitary-adrenal (HPA) axis function and behavior by synthetic glucocorticoids. Brain Res Rev 57: 586-595, 2008

5. Varcoe TJ, Boden MJ, Voultsios A, Salkeld MD, Rattanatray L and Kennaway DJ: Characterisation of the maternal response to chronic phase shifts during gestation in the rat: Implications for fetal metabolic programming. PLoS One 8: e53800, 2013.

6. Tiao MM, Huang LT, Chen CJ, Sheen JM, Tain YL, Chen CC, Kuo HC, Huang YH, Tang KS, Chu EW and Yu HR: Melatonin in the regulation of liver steatosis following prenatal glucocorticoid exposure. Biomed Res Int 2014: 942172, 2014.

7. Drake AJ, Raubenheimer PJ, Kerrigan D, McInnes KJ, Seckl JR and Walker BR: Prenatal dexamethasone programs expression of genes in liver and adipose tissue and increased hepatic lipid accumulation but not obesity on a high-fat diet. Endocrinology 151: $1581-1587,2010$

8. Tamashiro KL, Terrillion CE, Hyun J, Koenig JI and Moran TH: Prenatal stress or high-fat diet increases susceptibility to diet-induced obesity in rat offspring. Diabetes 58: 1116-1125, 2009.

9. Huang YH, Chen CJ, Tang KS, Sheen JM, Tiao MM, Tain YL, Chen CC, Chu EW, Li SW, Yu HR and Huang LT: Postnatal high-fat diet increases liver steatosis and apoptosis threatened by prenatal dexamethasone through the oxidative effect. Int J Mol Sci 17: 369, 2016.

10. Favero G, Lonati C, Giugno L, Castrezzati S, Rodella LF and Rezzani R: Obesity-related dysfunction of the aorta and prevention by melatonin treatment in ob/ob mice. Acta Histochem 115 783-788, 2013.

11. Govender J, Loos B, Marais E and Engelbrecht AM: Mitochondrial catastrophe during doxorubicin-induced cardiotoxicity: A review of the protective role of melatonin. J Pineal Res 57: 367-380, 2014

12. Tan DX, Manchester LC, Fuentes-Broto L, Paredes SD and Reiter RJ: Significance and application of melatonin in the regulation of brown adipose tissue metabolism: Relation to human obesity. Obes Rev 12: 167-188, 2011.

13. Hussein MR, Ahmed OG, Hassan AF and Ahmed MA: Intake of melatonin is associated with amelioration of physiological changes, both metabolic and morphological pathologies associated with obesity: An animal model. Int J Exp Pathol 88: 19-29, 2007.

14. Kitagawa A, Ohta Y and Ohashi K: Melatonin improves metabolic syndrome induced by high fructose intake in rats. J Pineal Res 52: 403-413, 2012.

15. García JJ, López-Pingarrón L, Almeida-Souza P, Tres A, Escudero P, García-Gil FA, Tan DX, Reiter RJ, Ramírez JM and Bernal-Pérez M: Protective effects of melatonin in reducing oxidative stress and in preserving the fluidity of biological membranes: A review. J Pineal Res 56: 225-237, 2014.

16. de Luxán-Delgado B, Caballero B, Potes Y, Rubio-González A, Rodríguez I, Gutiérrez-Rodríguez J, Solano JJ and CotoMontes A: Melatonin administration decreases adipogenesis in the liver of ob/ob mice through autophagy modulation. J Pineal Res 56: 126-133, 2014.

17. Celinski K, Konturek PC, Slomka M, Cichoz-Lach H, Brzozowski T, Konturek SJ and Korolczuk A: Effects of treatmen with melatonin and tryptophan on liver enzymes, parameters of fat metabolism and plasma levels of cytokines in patients with non-alcoholic fatty liver disease-14 months follow up. J Physio Pharmacol 65: 75-82, 2014

18. Hatzis G, Ziakas P, Kavantzas N, Triantafyllou A, Sigalas P, Andreadou I, Ioannidis K, Chatzis S, Filis K, Papalampros A and Sigala F: Melatonin attenuates high fat diet-induced fatty liver disease in rats. World J Hepatol 5: 160-169, 2013.

19. Zaitone S, Hassan N, El-Orabi N and El-Awady el-S: Pentoxifylline and melatonin in combination with pioglitazone ameliorate experimental non-alcoholic fatty liver disease. Eur J Pharmacol 662: 70-77, 2011.

20. National Research Council: Guide for the Care and Use of Laboratory Animals. 8th edition. National Acadamies Press. Washington, DC, 2011.
21. Hauser J, Feldon J and Pryce CR: Direct and dam-mediated effects of prenatal dexamethasone on emotionality, cognition and HPA axis in adult Wistar rats. Horm Behav 56: 364-375, 2009.

22. Lui CC, Hsu MH, Kuo HC, Chen CC, Sheen JM, Yu HR, Tiao MM, Tain YL, Chang KA and Huang LT: Effects of melatonin on prenatal dexamethasone-induced epigenetic alterations in hippocampal morphology and reelin and glutamic acid decarboxylase 67 levels. Dev Neurosci 37: 105-114, 2015.

23. Tain YL, Huang LT, Lin IC, Lau YT and Lin CY: Melatonin prevents hypertension and increased asymmetric dimethylarginine in young spontaneous hypertensive rats. J Pineal Res 49: 390-398, 2010

24. Lin TK, Huang LT, Huang YH, Tiao MM, Tang KS and Liou CW: The effect of the red wine polyphenol resveratrol on a rat model of biliary obstructed cholestasis: Involvement of anti-apoptotic signalling, mitochondrial biogenesis and the induction of autophagy. Apoptosis 17: 871-879, 2012.

25. Tiao MM, Wang FS, Huang LT, Chuang JH, Kuo HC, Yang YL and Huang YH: MicroRNA-29a protects against acute liver injury in a mouse model of obstructive jaundice via inhibition of the extrinsic apoptosis pathway. Apoptosis 19: 30-41, 2014.

26. Tiao MM, Lin TK, Kuo FY, Huang CC, Du YY, Chen CL and Chuang JH: Early stage of biliary atresia is associated with significant changes in 8-hydroxydeoxyguanosine and mitochondrial copy number. J Pediatr Gastroenterol Nutr 45: 329-334, 2007.

27. Nieto-Vazquez I, Fernández-Veledo S, Krämer DK, VilaBedmar R, Garcia-Guerra L and Lorenzo M: Insulin resistance associated to obesity: The link TNF-alpha. Arch Physiol Biochem 114: 183-194, 2008

28. Kwon $\mathrm{H}$ and Pessin JE: Adipokines mediate inflammation and insulin resistance. Front Endocrinol (Lausanne) 4: 71, 2013.

29. D'Souza K, Kane DA, Touaibia M, Kershaw EE, Pulinilkunnil T and Kienesberger PC: Autotaxin is regulated by glucose and insulin in adipocytes. Endocrinology 158: 791-803, 2017.

30. Rashid K, Das J and Sil PC: Taurine ameliorate alloxan induced oxidative stress and intrinsic apoptotic pathway in the hepatic tissue of diabetic rats. Food Chem Toxicol 51: 317-329, 2013.

31. Zhang Y and Yang JH: Activation of the PI3K/Akt pathway by oxidative stress mediates high glucose-induced increase of adipogenic differentiation in primary rat osteoblasts. J Cell Biochem 114: 2595-2602, 2013.

32. Davey MW, Stals E, Panis B, Keulemans J and Swennen RL: High-throughput determination of malondialdehyde in plant tissues. Anal Biochem 347: 201-207, 2005.

33. Del Rio D, Stewart AJ and Pellegrini N: A review of recent studies on malondialdehyde as toxic molecule and biological marker of oxidative stress. Nutr Metab Cardiovasc Dis 15: 316-328, 2005.

34. Candas D and Li JJ: MnSOD in oxidative stress response-potential regulation via mitochondrial protein influx. Antioxid Redox Signal 20: 1599-1617, 2014.

35. Diehl AM: Nonalcoholic steatosis and steatohepatitis IV. Nonalcoholic fatty liver disease abnormalities in macrophage function and cytokines. Am J Physiol Gastrointest Liver Physiol 282: G1-G5, 2002.

36. Tacke $\mathrm{F}$, Luedde $\mathrm{T}$ and Trautwein $\mathrm{C}$ : Inflammatory pathways in liver homeostasis and liver injury. Clin Rev Allergy Immunol 36: 4-12, 2009.

37. Canbakan B, Senturk H, Canbakan M, Toptas T, Tabak O, Balci H, Olgac V and Ozbay G: Is alanine aminotransferase level a surrogate biomarker of hepatic apoptosis in nonalcoholic fatty liver disease? Biomark Med 4: 205-214, 2010

38. Guha M, Maity P, Choubey V, Mitra K, Reiter RJ and Bandyopadhyay $\mathrm{U}$ : Melatonin inhibits free radical-mediated mitochondrial-dependent hepatocyte apoptosis and liver damage induced during malarial infection. J Pineal Res 43: 372-381, 2007.

39. Cruz A, PadilloFJ, Torres E, Navarrete CM, Muñoz-Castañeda JR, Caballero FJ, Briceño J, Marchal T, Túnez I, Montilla P, et al: Melatonin prevents experimental liver cirrhosis induced by thioacetamide in rats. J Pineal Res 39: 143-150, 2005.

40. Padillo FJ, Cruz A, Navarrete C, Bujalance I, Briceño J, Gallardo JI, Marchal T, Caballero R, Túnez I, Muntané J, et al: Melatonin prevents oxidative stress and hepatocyte cell death induced by experimental cholestasis. Free Radic Res 38: 697-704, 2004.

41. Heeba GH and Morsy MA: Fucoidan ameliorates steatohepatitis and insulin resistance by suppressing oxidative stress and inflammatory cytokines in experimental non-alcoholic fatty liver disease. Environ Toxicol Pharmacol 40: 907-914, 2015. 
42. Liu Y, Song A, Zang S, Wang C, Song G, Li X, Zhu Y, Yu X, Li L, Wang Y and Duan L: Jinlida reduces insulin resistance and ameliorates liver oxidative stress in high-fat fed rats. J Ethnopharmacol 162: 244-252, 2015.

43. Jiang Y, Chen L, Wang H, Narisi B and Chen B: Li-Gan-Shi-LiuBa-Wei-San improves non-alcoholic fatty liver disease through enhancing lipid oxidation and alleviating oxidation stress. J Ethnopharmacol 176: 499-507, 2015.

44. Jorgensen SB, O'Neill HM, Sylow L, Honeyman J, Hewitt KA, Palanivel R, Fullerton MD, Öberg L, Balendran A, Galic S, et al: Deletion of skeletal muscle SOCS3 prevents insulin resistance in obesity. Diabetes 62: 56-64, 2013.

45. Yang G, Badeanlou L, Bielawski J, Roberts AJ, Hannun YA and Samad F: Central role of ceramide biosynthesis in body weight regulation, energy metabolism, and the metabolic syndrome. Am J Physiol Endocrinol Metab 297: E211-E224, 2009.
46. Shi H, Cave B, Inouye K, Bjørbaek C and Flier JS: Overexpression of suppressor of cytokine signaling 3 in adipose tissue causes local but not systemic insulin resistance. Diabetes 55: 699-707, 2006.

47. Buzzelli MD, Navaratnarajah M, Ahmed T, Nagarajan M, Shumate ML, Lang CH and Cooney RN: Nuclear factor kappaB mediates the inhibitory effects of interleukin-1 on growth hormone-inducible gene expression. J Trauma 64: 1427-1436, 2008.

48. Torisu T, Sato N, Yoshiga D, Kobayashi T, Yoshioka T, Mori H, Iida $\mathrm{M}$ and Yoshimura A: The dual function of hepatic SOCS3 in insulin resistance in vivo. Genes Cells 12: 143-154, 2007. 